\title{
Evoked potentials and sensation
}

D. REGAN 2,3

UNIVERSITY OF KEELE

\begin{abstract}
Objective physiological measurements (evoked potentials) were compared with the corresponding psychophysical observations for human Ss. Discrepancies were found between the amplitude of the steady-state evoked potential (EP) and the sensations of flicker produced by visual stimuli of different modulation depths. Poor correlations were also found between subjective De Lange curves and amplitude vs frequency curves for the EP under conditions of chromatic adaptation. It is suggested that two classes of EP can be distinguished; one correlates well and the other correlates poorly with sensation.
\end{abstract}

In attempts to identify the mechanisms which underlie perception, the technique of evoked potential (EP) recording has an important advantage: the fact that EPs can be recorded without discomfort from human Ss, so that objective measurements of brain activity can easily be correlated both with psychophysical measurements and with subjective reports of sensations.

The present report considers two experiments designed to make use of this advantage. They deal, respectively, with aspects of the EP in relation to (1) flicker modulation and (2) De Lange curves.

\section{METHODS}

The light source of the visual stimulator (Fig. 1) was a 500-W Xenon arc. The stimulus intensity was sinusoidally modulated at a frequency $F$ between 2 and $60 \mathrm{cps}$ by rotating and fixed polaroids which were driven through gears by a high-precision variable speed motor. A modulated beam of one color could be superposed on a steady adapting beam of the same or of a different color by means of a two-beam optical system (Kelly, 1961). Monochromatic beams were obtained with interference filters of about $10 \mathrm{~nm}$ half-width, and the stimulus intensity was altered by the use of neutral density filters. This optical arrangement enabled the modulation depth of the stimulus to be varied without changing the mean stimulus intensity. The $S$ was seated in a soundproof

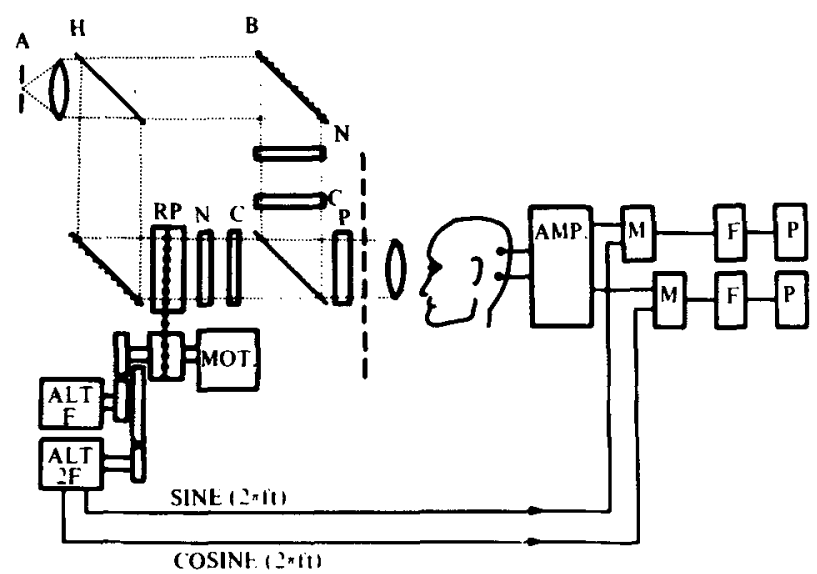

Fig. 1. Visual stimulator and Fourier analyzer. A-500-W Xenon arc lamp; B-mirror; $\mathbf{H}$-half silvered mirror; $\mathbf{N}$-neutral density filters; $\mathbf{C}$-interference flter; RP-rotating polaroid; P-fixed polaroid; MOT-high-stability, variable speed motor; ALTF-alternator geared to fundamental frequency; ALT 2F-alternator geared to second harmonic frequency; AMP-EEG amplifier; M-Hall effect multiplier; F-low-pass fitter; P-recording potentiometer.

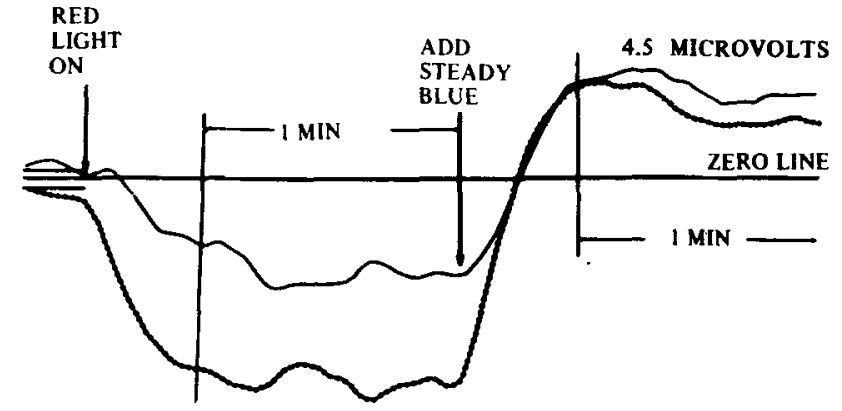

10 MICROVOLTS

Fig. 2. Fourier analyzer used to give an on-ine demonstration of the effect on the amplitude and phase of the fundamental component of the EP of adding steady blue light to a red stimulus light. Red stimulus of mesn retinal illumination 1300 trolands and $60 \mathrm{deg}$ subtense. Blue light of 1300 trolunds and $60 \mathrm{deg}$ subtenac. Bipolar recordings made between Electrodes 2 and 4 (Fig. 3). Deflections of pens from zero line $\left(\theta_{1}\right.$ and $\left.\theta_{2}\right)$ give amplitude and phase of fundamental component of EP. Adding steady light halves amplitude and changes phase by about $150 \mathrm{deg}$.

chamber in order to avoid both the complicating effects of auditory distraction and contamination of the EP by auditory evoked potentials. The stimulus ( whose plane of polarization was unmodulated) was presented in Maxwellian view through an exit pupil formed by an image which was located at the center of the S's natural pupil. Fixation was improved by the use of a bite bar.

The EEG was analyzed in two ways:

(1) A cross-correlator extracted a specified harmonic component of the EP from the EEG and displayed a "running average" of the pliase and amplitude of the component (Fig. 1). The principle of this device has been described previously (Regan, 1966a). The EEG was separately multiplied by sine and cosine waveforms generated by an alternator which was driven at a multiple or submultiple of the stimulus modulation frequency. The harmonic component to be extracted was determined by the gear ratio between the alternator and the modulating polaroid. After low-pass filtering, the sine and cosine products were displayed on a two-pen recording potentiometer, which gave deflections $\theta_{1}$, and $\theta_{2}$. These deflections indicated the running averages of the amplitude $\left(\alpha \sqrt{ } \theta_{1}^{2}+\theta_{2}^{2}\right)$ and phase [ $\left.\tan ^{-1}\left(\theta_{1} / \theta_{2}\right)\right]$ of the harmonic component which had initially been specified by the choice of gears. The equipment was allowed to run for about $30 \mathrm{sec}$ before the stimulus was switched on, in order to assess the noise level in the "no-light" conditions. Running average measurements were made over 1-3 min, mostly in the "dynamic" or "average" steady-state condition (Regan, 1966a) which developed some 12-20 sec after stimulus onset.

It was often found useful to employ the running average technique in such a way that the results of changing an individual stimulus parameter were displayed almost immediately. One result of pairing observations in this manner was to minimize the effect of variability between one observation and another. The method is illustrated in Fig. 2, which demonstrates an effect of chromatic adaptation on the EP. For this demonstration, the $S$ was stimulated by a red light, modulated at $16 \mathrm{cps}$, until the steady-state response had been established for about $1 \mathrm{~min}$. Steady blue light was then added to the modulated red beam, and the visual system was allowed to reach a second steady-state condition 
which was then maintained for about $1 \mathrm{~min}$. Figure 2 illustrates how it could be seen on-line that one result of adding the steady blue adapting light was that the amplitude of the EP immediately fell by about $50 \%$.

(2) In some experiments the EEG was also analyzed with an averaging computer (CAT 400B) which recorded average waveforms between electrodes 2-1, 2-3, and 2-4 (Fig. 3).

\section{RESULTS}

Experiment 1: The Perception of Flicker and the Amplitude of the EP.

A stimulus of constant mean intensity of about 3900 trolands was sinusoidally modulated at a constant frequency, and the modulation depth was gradually increased from zero to $100 \%$. The $\mathrm{S}$ reported that flicker grew progressively more prominent. On the other hand, although the fundamental component of the steady-state EP rose approximately linearly until a modulation depth of about $10 \%$ was reached, it subsequently levelled off in a manner suggestive of an automatic gain control (Fig. 3 ). In some expr-iments the amplitude of the EP fell off at high depths of moduration. Average EP waveforms which were recorded with the CAT computer were found to be particularly pure sinusoids. This means that Fig. 3 also gives a fair description of the behavior of the averaged EP. Similar saturation phenomena have previously been reported by vander Tweel and Lunel (1965), and by Spekreijse (1966).

\section{Discussion}

If the amplitude of the EP correlated with perceived flicker over the full effective range of modulation depths, then each $S$ should have reported a subjective effect which correlated with the "knee" in their EP curves. There were no such reports, however.

One explanation for this disparity between EP and perception might be that there is a fork in the visual pathway, and that one branch relates to the generation of the EP and the other branch relates to the mechanisms of flicker perception. The steady-state EP might be only indirectly related to the perception of flicker, or it might merely be contaminated by mechanisms other than those of the perception of flicker. One example of this latter possibility may be the mechanisms of lateral interaction which seem to be concerned in the color effects in the EP which occur when stimuli are modulated at about 16 cps (Regan, 1968a).

If the visual pathway does branch, limits can be set on the position of the fork by comparing the saturated values of EP amplitude for monocular and binocular stimulation (Spekreijse, 1966). For the stimulus of Fig. 3 the saturated value of the amplitude of the EP to binocular stimulation was found to be

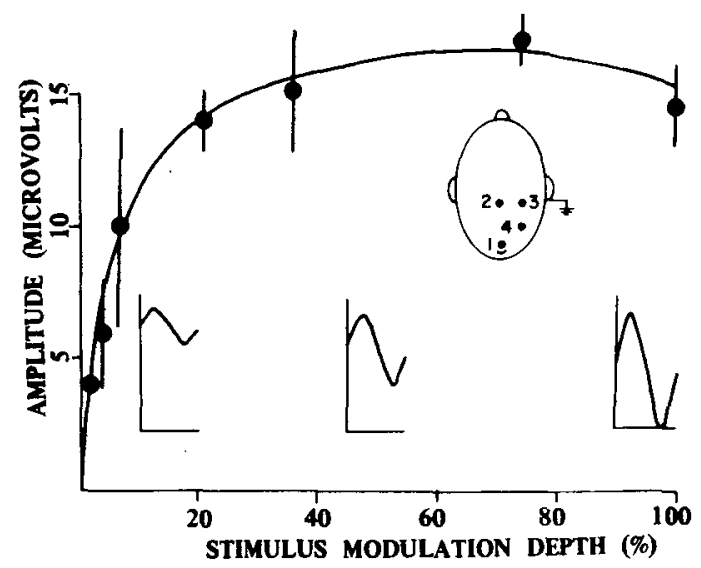

Fig. 3. Amplitude of fundamental component of steady-state EP (microvolts) vs stimulus modulation depth (\%). The stimulus field was of 14 deg subtense, wavelength $639 \mathrm{~mm}$, with a dark surround. The mean retinal illumination was about 3900 trolands and was sinusoidally modulated at 16 cps. Bipolar recordings were made between Electrodes 2 and 4. Subject D.R. roughly equal to the sum of the amplitudes of the EPs to left- and right-eyed monocular stimulation. This suggests that the fork between the pathway relating to the perception of flicker and the pathway relating to the generation of the EP occurs before the stage at which the signals from the two eyes unite.

An additional technical point is that saturation behavior indicates that a measure of simplification in some EP studies would result if stimuli were initially restricted to the presaturation region, where at least one of the elements of the EP system is approximately linear. This calls for small-signal conditions, that is, for the use of modulated light rather than flashes on a dark background.

Experiment 2: De Lange Curves for Perceived Flicker, and the EP.

Notable advances in the understanding of the perception of flicker followed De Lange's use of sinusoidally modulated light in his systematic investigation of the threshold for the perception of flicker. He measured the modulation depth $m(m=$ half the peak-to-peak percentage variation of intensity) for which flicker could just be perceived (De Lange, 1957). His choice of modulated light as a stimulus enabled him to study the individual effects of the intensity and the frequency of the stimulus on the threshold modulation depth. De Lange presented his results in the form of a plot of the reciprocal of the threshold value of $\mathrm{m}$ vs flicker frequency. This De Lange curve describes the attentuation characteristics of the visual pathways which relate to the perception of flicker. De Lange found an attenuation of about $18 \mathrm{~dB} /$ octave for the high frequency region and a maximum sensitivity to flicker at about $10 \mathrm{cps}$. This maximum was most prominant at high retinal illuminations.

Kelly subsequently observed that the low frequency part of the De Lange curve was dependent on the spatial characteristics of the stimulus field (Kelly, 1959), and went on to extend De Lange's work on the effects of color on the threshold by superposing a flickering stimulus light of one color on a steady adapting background of a different color. His aim in using chromatic adaptation was to selectively emphasize the characteristics of long, medium, and short wavelength components of the De Lange curve (Kelly, 1962). The curves for some Ss had a subpeak at $47 \mathrm{cps}$ characteristic of a "blue" system, a subpeak at 10-15 cps characteristic of a "green" system, and a subpeak at 20-30 cps characteristic of a "red" system. On the other hand, the high-frequency part of the De Lange curve was comparatively little affected by chromatic adaptation. Kelly suggested that the frequencies of the subpeaks were governed by the different time constants of the short, medium, and long wavelength mechanisms in the visual pathways.

This evidence for a link between the physiological mechanisms of color vision and the subpeaks of the De Lange curve suggested that Kelly's experiments might profitably be extended to include objective electrophysiological observations. Accordingly, the De Lange curve and the amplitude vs frequency characteristic of the EP have been measured under similar stimulus conditions (Fig. 4). The psychophysical and electrophysiological data were then compared in order to find whether there were subpeaks in the EP curves which corresponded to Kelly's subpeaks in the De Lange curves, and, if so, whether these electrophysiological subpeaks showed a similar dependence on color to the psychophysical subpeaks.

The stimulus field was of $60 \mathrm{deg}$ subtense and was sinusoidally modulated in intensity. The color of the stimulus was red, blue, or modulated red superposed on steady blue. The EEG was analyzed both with an averaging computer and with the cross-correlator described above, and the amplitude of the fundamental component of the EP, and the peak-to-peak amplitude of the averaged response waveform measured. Four Ss with normal color vision (Ishihara test) were used.

In Fig. 4 the EP and De Lange curves are compared for modulated red light, and for modulated red light superposed on a steady blue adapting background. It can be seen in Fig. 4 that there were clear subpeaks in the EP curves, and that the relative amplitudes of these subpeaks were strongly influenced by the 


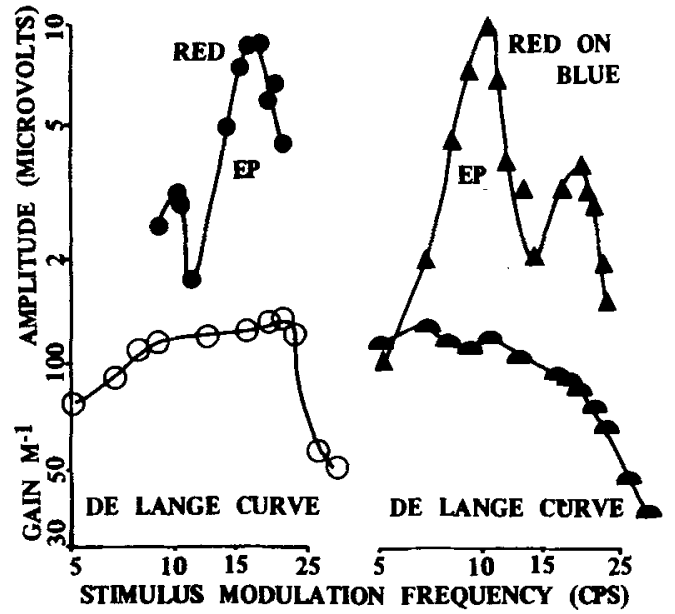

Fig. 4. Comparison of the attenuation characteristics for the visual pathway relating to the perception of flicker (De Lange curves), and for the visual pathway relating to the generation of the EP. Upper curves are plots of the amplitude of the fundamental component of the steady-state EP vs the stimulus modulation frequency when the stimulus luminance was modulated to a depth of $33 \%$. The lower curves ere plots of the reciprocal of the modulation depth at the threshold of perception of flicker vs stimulus modulation frequency (De Lange curves). The left hand curves are for a red stimulus of wavelength $639 \mathrm{~nm}$, and the right hand curves are for a modulated red stimulus superposed on a steady blue light of wavelength $460 \mathrm{~nm}$. The subtense of the stimulus field was $60 \mathrm{deg}$, and the mean retinal illumination about 3900 trolmds. Subject D.R.

color of the steady adapting light. On the other hand, this $S$ did not show corresponding subpeaks in the psychophysical curves (Fig. 4). Furthermore, the changes in the amplitude of the subpeaks of the EP curves which were produced by chromatic adaptation are not paralleled by changes in the De Lange curves.

The EP data of Fig. 4 were obtained with a stimulus modulation depth of $33 \%$ which, except for the high frequency region, is considerably higher than the threshold values of the De Lange data. However, no significant differences were found in the shape of the EP curves when the stimulus modulation depth was reduced to $10 \%$, which was well below the level which produced saturation of the EP, and was much nearer to the value for the psychophysical threshold. A similar lack of correspondence between EP data and the De Lange curves was found for the other Ss used in this experiment.

The above account is restricted to stimulus frequencies below about $20 \mathrm{cps}$, in a frequency region where the steady-state EP has been found to depend on the color of the stimulus (Regan, 1966b, 1968a). Correlations between perception and the EP have also been investigated for higher stimulus modulation frequencies, at which the De Lange curve is little affected by stimulus field size or color (Kelly, 1962). Observations were made at stimulus modulation depths which were either below the threshold for perceived flicker, or did not greatly exceed threshold, and a high frequency peak was found in the amplitude vs frequency curve for the EP (Regan, 1968b). The maximum amplitude of this peak occurred at a frequency so high that flicker could not be perceived (van der Tweel \& Lunel, 1965; Regan, 1968b). No corresponding peak was found in the psychophysical curve. This indicates that a neural representation of flicker, which is presumably present at a high level in the central nervous system, is not effectively available to the mechanisms of perception.

\section{Discussion}

If the amplitude of the EP reflected the neural representation of flicker which underlies perception, then it might be expected that the attenuation vs frequency characteristic for the EP would match the De Lange curve. However, for stimulus frequencies between 7 and 20 cps clear differences were found between the two curves. At higher frequencies of stimulation, too, very little correlation between flicker perception and the EPs of some Ss was found, both in the high frequency part of the De Lange curve and beyond the fusion point for perceived flicker. This means that the pathway relating to the perception of flicker has a different attenuation vs frequency characteristic from the pathway relating to the generation of the EP, and provides evidence in addition to that of Experiment 1 to suggest that there is a fork in the visual pathway, where one branch relates to the perception of flicker and the other branch relates to the generation of the EP.

One explanation of this disagreement between psychophysical and electrophysiological data might be that the methods used in the experiments described above could detect only those electrophysiological responses which were locked to the stimulus. In gross electrode work it is technically convenient to record only those signals which are locked to the stimulus. There is no justification, however, for assuming that stimulus-locked responses are the only responses which are likely to correlate with flicker perception. On the contrary, there are grounds for the view that responses which are not locked to the stimulus might be correlated with flicker perception. For example, a prominent feature of the perception of flicker for stimulus frequencies above about $10 \mathrm{cps}$ is that the perceived frequency of flicker does not necessarily correspond to the stimulus frequency, nor to one of its harmonics (Shipley, 1964), and, furthermore, the perceived flicker frequency is strongly influenced by simultaneous acoustic stimulation (Knox, 1945). Indirect evidence for this possibility is offered by a report that although the firing patterns of individual fibers of the optic rerve were influenced by the flickering of a visual stimulus, the activity was not locked to the stimulus (Fukada et al, 1966).

\section{CONCLUSIONS}

The experimental methods described in this paper lend themselves to quantitative specification of both stimulus and response. As a result, it is particularly easy to see the relationships between features of the stimulus and the steady-state evoked potential, and in some Ss it is possible to identify stimulus features from the evoked potentials alone.

On the other hand, the correlations between features of the steady-state evoked potential and sensation were found to be remarkably poor. It is always possible that a different feature of the evoked potential, such as topography for example, would correlate more closely with sensation than the curves of amplitude, phase, and harmonic distortion which were in fact measured. One possible explanation for the results described in this paper is that the physically very simple stimuli which were used in this investigation are so simple that they are inappropriate stimuli for the physiological mechanisms which underlie perception. It is intriguing that the converse of this has experimental support, in that good correlations have been reported between features of the evoked potential and the visual sensations which are produced by physically rather complex stimuli (MacKay \& Fiorentini, 1966; MacKay, 1968).

\section{APPENDIX \\ Redated Aspects of Evoked Potential Quantification}

The potentials evoked by flashes and by step and ramp stimuli can provide a description of transient response characteristics of the visual system. A second viewpoint onto the evoked potential visual system may be given by the steady-state response plots of amplitude vs modulation frequency and phase vs modulation frequency. A third and different approach is to investigate the influence on the evoked potential of the temporal structure of the stimulus (MacKay \& Fiorentini, 1966).

If it is decided to study the steady-state responses of the visual system there are a number of reasons for using sinusoidally modulated stimuli.

In many experiments short flashes or square waves have been used to obtain the amplitude and phase characteristics for steady-state EPs, and in a large proportion of these experiments the effective stimulus frequency was not known. The reason for this is that a repetitive flash stimulus can be regarded as a compound of several harmonics of the repetition frequency. In the frequency-dependent human visual system (van der Tweel \& Lunel, 1965; Regan, 1966a) the physiologically most effective stimulus frequency need not necessarily be the lowest of these multiples of the flash frequency. This 
means that the steady-state responses which are evoked by flash stimulation do not give a basic description of the visual system; they do not relate in any simple manner to the results of stimulation by other waveforms. It is at best difficult to calculate the amplitude $v$ frequency and phase vs frequency curves for the system from such flash data.

These difficulties in obtaining the amplitude vs frequency and phase vs frequency characteristics for steady-state EPs can be avoided by the use of sinusoidally modulated stimuli (van der Tweel \& Lunel, 1965; Regan, 1966a). Modulated light has the advantage over flashes that the stimulus parameters of mean intensity and modulation depth can be separately varied. This is important, since these two parameters affect the EP quite differently (Spekreijse, 1966). A harmonically simple waveform (the extreme case being sinusoidal) lends itself to clear quantitative description, and enables the response of the visual system at different stimulus frequencies to be investigated without ambiguity. I addition, any distortions found in the EP waveforms can be confidently attributed to the visual system in terms of "what sort of distortion?" and "how much distortion?". The value of quantitative data on distortion has been demonstrated by Spekreijse and van der Tweel (1965) and Spekrejise (1966) who showed that these data may give clues to mechanisms in the visual system. None of this is possible, and, furthermore, "distortion" loses meaning when flashes or square waves are used to study the steady-state transfer function of the visual system.

Clynes et al (1964) have pointed out that in some nonlinear physiological systems, Fourier analysis by itself does not give a complete description of the steady-state response, since in these systems a frequency-dependent dc shift is generated in addition to the oscillatory response. Furthermore, transient and steady-state methods of investigation may reveal different aspects of the functioning of a nonlinear system such as the human visual system (van der Tweel \& Lunel, 1965) rather than merely provide different representations of the same data. In a wide range of experiments, therefore, neither intermittent nor repetitive stimulation by itself can give a complete description of the system; often neither an averaging computer nor a Fourier analyzer by itself can provide the experimenter with an adequate description of the response waveform evoked by repetitive stimulation. This indicates that these techniques should all be brought to bear on EP problems whenever the experimental design allows.

\section{REFERENCES}

CLYNES, M., KOHN, M., \& LIFSHITZ, K. Dynamic and spatial behavior of light evoked potentials, their modification under hypnosis, and on-line correlation in relation to rhythmic components. Annals of the New York Academy of Sciences, 1964, 112, 468-508.

DE LANGE, H. Attenuation characteristics and phase-shift characteristics of the human fovea-cortex system in relation to flicker fusion phenomena. Thesis, 1957, Delft.
FUKADA, Y., MOTOKAWA, K., NORTON, A. C., \& TASAKI, K. Functional significance of conduction velocity in the transfer of flicker information in the optic nerve of the cat. Journal of Neurophysiology, 1966, 29, 698-714.

KELLY, D. H. Effects of sharp edges in a flickering field. Journal of the Optical Society of America, 1959, 49, 730-732.

KELLY, D. H. Visual signal generator. Review of Scientific Instruments, $1961,32,50-55$.

KELLY, D. H. Visual responses to time-dependent stimuli. IV. Effects of chromatic adaptation. Journal of the Optical Society of America, 1962, $52,940-947$.

KNOX, G. W. Investigations of flicker and fusion. IV. The effect of auditory flicker on the pronouncedness of visual flicker. Journal of General Psychology, 1945, 33, 145-154.

MacKAY, D. M. Evoked potentials reflecting interocular and monocular suppression. Nature, 1968, 217, 8183.

MacKAY, D. M., \& FIORENTINI, A. Evoked potentials correlated with a visual anomaly. Nature, 1966, 209, 787-789.

REGAN, D. Some characteristics of average steady-state and transient responses to modulated light. Electroencephalography \& Clinical Neurophysiology, 1966a, 20, 238-248.

REGAN, D. An effect of stimulus color on average steady-state potentials evoked in man. Nature, 1966b, 210, 1056-1057.

REGAN, D. Chromatic adaptation and steady-state evoked potentials. Vision Research, 1968a, 8, 149-158.

REGAN, D. A high frequency mechanism which underlies visual evoked potentials. Electroencephalography \& Clinical Neurophysiology, 1968b, 25, 231-237.

SHIPLEY, T. Auditory flutter-driving of visual flicker. Science, 1964, 145, 1328-1330.

SPEKREUSE, H. Analysis of EEG responses in man. Thesis, 1966, Amsterdam.

SPEKREIJSE, H., \& VAN DER TWEEL, L. H. Linearisation of evoked responses to sine wave-modulated light by noise. Nature, 1965, 205, 913.

VAN DER TWEEL, L. H., \& LUNEL, H. F. E. VERDUYN. Human visual responses to sinusoidally modulated light. Electroencephalography \& Clinical Neurophysiology, 1965, 18, 587-598.

\section{NOTES}

1. The support of the Medical Research Council is gratefully acknowledged.

2. Address: Department of Communication, University of Keele, Keele, Staffordshire, England.

3. My thanks are due to Robert F. Cartwright for his skillful construction of equipment and for many contributions to its design. I am grateful to Frances Shaw, Jeffrey Sedgeley, David Whitebread, and other volunteer Ss for their patience.

(Accepted for publication A ugust 8, 1968.) 